SENSORS

pubs.acs.org/acssensors

\title{
SERS-Based Molecularly Imprinted Plasmonic Sensor for Highly Sensitive PAH Detection
}

\author{
Alexander Castro-Grijalba, Verónica Montes-García, María José Cordero-Ferradás, Eduardo Coronado, \\ Jorge Pérez-Juste, ${ }^{*}$ and Isabel Pastoriza-Santos*
}

Cite This: ACS Sens. 2020, 5, 693-702

Read Online

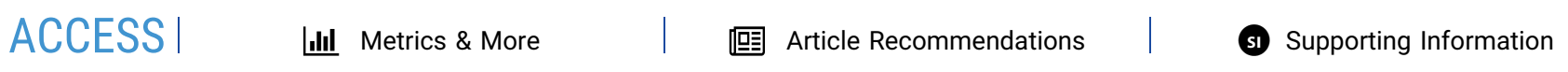

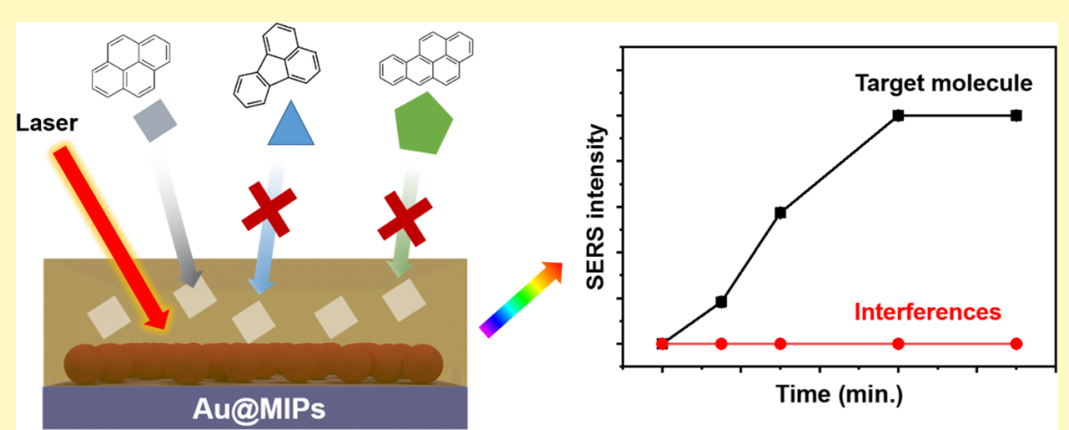

ABSTRACT: A novel hybrid plasmonic platform based on the synergetic combination of a molecularly imprinted polymer (MIP) thin film with Au nanoparticle (NPs) assemblies, noted as Au@MIP, was developed for surface-enhanced Raman scattering (SERS) spectroscopy recognition of polycyclic aromatic hydrocarbons (PAHs). While the MIP trapped the PAH close to the Au surface, the plasmonic NPs enhanced the molecule's Raman signal. The Au@MIP fabrication comprises a two-step procedure, first, the layer-bylayer deposition of Au NPs on glass and their further coating with a uniform MIP thin film. Profilometry analysis demonstrated that the thickness and homogeneity of the MIP film could be finely tailored by tuning different parameters such as prepolymerization time or spin-coating rate. Two different PAH molecules, pyrene or fluoranthene, were used as templates for the fabrication of pyrene- or fluoranthene-based Au@MIP substrates. The use of pyrene or fluoranthene, as the template molecule to fabricate the $\mathrm{Au} @ \mathrm{MIP}$ thin films, enabled its ultradetection in the $\mathrm{nM}$ regime with a 100 -fold improvement compared with the nonimprinted plasmonic sensors (Au@NIPs). The SERS data analysis allowed to estimate the binding constant of the template molecule to the MIP. The selectivity of both pyrene- and fluoranthene-based Au@MIPs was analyzed against three PAHs of different sizes. The results displayed the important role of the template molecule used for the Au@MIPs fabrication in the selectivity of the system. Finally, the practical applicability of pyrene-based Au@MIPs was shown by performing the detection of pyrene in two real samples: creek water and seawater. The design and optimization of this type of plasmonic platform will pave the way for the detection of other relevant (bio)molecules in a broad range of fields such as environmental control, food safety, or biomedicine.

KEYWORDS: molecularly imprinted polymers, hybrid nanostructures, plasmonic sensors, SERS, polycyclic aromatic hydrocarbons, environmental analysis

\section{INTRODUCTION}

Molecularly imprinted polymers (MIPs) are tailor-made polymers that are prepared in the presence of a template entity (atom, ion, molecule, complex, or a molecular, ionic or macromolecular assembly, including micro-organisms). ${ }^{1}$ After the subsequent removal of the template molecules, the vacant spaces will act as recognition sites with suitable shape, size, and functional groups for the detection/separation of a target analyte (template). MIPs are hence three-dimensional supramolecular smart materials with remarkable molecular recognition properties. ${ }^{1}$ Compared to other recognition systems, MIPs have become attractive in many fields such as purification and separation, ${ }^{2}$ chemo/biosensing, ${ }^{3-6}$ artificial antibodies, ${ }^{7}$ drug delivery, ${ }^{8}$ and catalysis and degradation, ${ }^{9}$ owing to their high physical stability, straightforward and lowcost preparation, and remarkable robustness. ${ }^{10}$ However, the use of MIPs for sensing applications still has some drawbacks such as converting the binding event into a measurable and quantifiable signal, and hence, it could be cumbersome to perform a multiplex analysis.

Received: September 25, 2019

Accepted: March 5, 2020

Published: March 5, 2020 
Surface-enhanced Raman scattering (SERS) spectroscopy is a powerful analytical technique for ultrasensitive detection of molecules. It is based on the enhancement of the characteristic inelastic Raman scattering signals of an analyte when it is in close proximity to a plasmonic metal surface ( $\mathrm{Au}$ and $\mathrm{Ag}$, mainly). ${ }^{11,12}$ The unique spectral features with narrow peaks make SERS ideal for multiplexing. Moreover, SERS allows portability and fast analysis without the need for sample pretreatment. ${ }^{13}$ However, SERS sensing still has some limitations as many analytes, including the polycyclic aromatic hydrocarbons (PAHs), lack metal-affinity functional groups which limit their effective detection. In those cases, SERS detection is performed by capturing the molecule through the proper functionalization of the metal surface. ${ }^{14-16}$ Therefore, the combination of MIPs with plasmonic nanostructures could lead to a strong synergistic effect; where the MIP will act as a recognition and trapping site for the target analyte and the plasmonic nanoparticles (NPs) will play the important role of enhancing the molecule Raman signal. The outstanding optical properties of $\mathrm{Au}$ NPs have shown great potential for the development of plasmonic-based Au@MIP hybrid sensors with promising capabilities in terms of sensitivity, selectivity, and reliability. ${ }^{17}$ In fact, several attempts have been made to develop Au@MIP substrates as optical, ${ }^{18}$ electrochemical, ${ }^{19}$ and gravimetric sensors. ${ }^{20}$ On the other hand, MIPs combined with SERS have been successfully employed for the analysis of pesticides, ${ }^{21}$ antibiotics, ${ }^{22}$ proteins, ${ }^{23}$ explosives, ${ }^{24}$ vitamins, ${ }^{25}$ or toxins. ${ }^{26}$ Nevertheless, in most cases, the strategy implies two separate steps: (1) MIP extraction and preconcentration of the analyte and (2) SERS detection. Alternatively, just a few works have been reported combining both elements, MIPs and plasmonic NPs, in a single platform. ${ }^{24,27}$ For instance, Holthoff et al. proposed a micron-size MIP coating on Klarite substrates for the SERS-based detection of 2,4,6-trinitrotoluene. ${ }^{24}$ Alternatively, Liz-Marzán and co-workers reported the use of multibranched gold-silica-MIP ( $\left.\mathrm{Au} @ \mathrm{mSiO}_{2} @ \mathrm{MIP}\right)$ coreshell NPs, for the ultrasensitive SERS detection of enrofloxacin. ${ }^{27}$

PAHs are an important class of environmental pollutants that are produced during incomplete combustion of materials such as oil, coal, petrol, or wood. Hence, they can be found in the atmosphere, soil, natural waters, and even in food consumed by humans. ${ }^{28-32}$ These issues make relevant the development of sensitive analytical techniques for their detection. Gas and liquid chromatography-mass spectrometry is perhaps the main technique for $\mathrm{PAH}$ analysis, ${ }^{33}$ which presents important drawbacks as sample treatment and hence a long analysis time or lack of portability. It should be pointed out that MIPs have been used to preconcentrate PAHs for HPLC and GC analysis. ${ }^{34,35}$ Thus, the development of new hybrid plasmonic materials based on MIPs as SERS substrates open the possibility to meet the current requirements of reaching higher sensitivity, faster analysis, and eventually portability. To the best of our knowledge, no MIPs-SERS substrates for PAHs detection have been reported yet.

Herein, we report the fabrication of a hybrid plasmonic SERS chemical sensor by combining in the same platform the molecular recognition capability of MIPs with the highperformance SERS detection of Au NP assemblies. Although the plasmonic platform was fabricated through the layer-bylayer (LbL) deposition of Au NPs, a uniform MIP thin film was deposited by spin-coating. The fabrication of the polymer film required the analysis of polymerization conditions (prepolymerization time and spin-coating rate) of methacrylic acid (MAA) and divinylbenzene (DVB) in the presence of a template. Two different PAHs, pyrene and fluoranthene, were employed independently as templates to fabricate pyrenebased or fluoranthene-based Au@MIP thin films, respectively. We investigated the sensing capabilities of each Au@MIP, as well as their selectivity when binary or ternary mixtures of PAHs (pyrene, fluoranthene, and/or benzo[a]pyrene) were used. Finally, the performance of pyrene-based Au@MIPs was assessed for the detection of pyrene in spiked real matrices (creek water and seawater).

\section{EXPERIMENTAL SECTION}

Materials. Tetrachloroauric(III) acid trihydrate $\left(\mathrm{HAuCl}_{4} \cdot 3 \mathrm{H}_{2} \mathrm{O}\right.$, $99.995 \%)$, trisodium citrate dihydrate, poly(diallyldimethylammonium chloride) (PDDA, 20 wt \% in $\mathrm{H}_{2} \mathrm{O}$, average $M_{\mathrm{w}}$ 200,000-350,000), poly(sodium 4-styrenesulfonate) (PSS, $\left.M_{\mathrm{w}} 70,000\right)$, hydrogen peroxide $\left(\mathrm{H}_{2} \mathrm{O}_{2}, 28 \%\right)$, sulfuric acid $\left(\mathrm{H}_{2} \mathrm{SO}_{4}, 98 \%\right)$, sodium chloride $(\mathrm{NaCl}, 99 \%)$, MAA (99\%), DVB (80\%), 2,2'-azobis(2-methylpropionitrile) (AIBN), fluoranthene (98\%), and benzo-[a]-pyrene (96\%) were supplied by Sigma-Aldrich. Pyrene (99\%) was supplied by Merck. N,N-dimethylformamide (DMF) was supplied by Fluka. Milli-Q grade water was used in all preparations.

Synthesis of Au NPs. Citrate-stabilized Au NPs $(\sim 60 \mathrm{~nm}$ in diameter) were synthesized following a seeded growth method previously reported by Bastús et al. ${ }^{36}$ In brief, $150 \mathrm{~mL}$ of an aqueous solution of $2.2 \mathrm{mM}$ trisodium citrate was heated to boiling under vigorously stirring. After $15 \mathrm{~min}, 1 \mathrm{~mL}$ of $25 \mathrm{mM} \mathrm{HAuCl}_{4}$ in water was injected into the boiling solution. After $10 \mathrm{~min}$, the reaction mixture was cooled down to $90{ }^{\circ} \mathrm{C}$ and subsequently, another injection of $1 \mathrm{~mL}$ of $25 \mathrm{mM} \mathrm{HAuCl}_{4}$ in water was performed. Another addition was repeated $30 \mathrm{~min}$ later. The mixture was allowed to react for $30 \mathrm{~min}$ and then, $55 \mathrm{~mL}$ were extracted from the reaction mixture. Subsequently, $53 \mathrm{~mL}$ of water and $2 \mathrm{~mL}$ of $60 \mathrm{mM}$ sodium citrate (in water) were added. The resulting solution was used as Au seeds. The process was repeated six times to yield $60 \mathrm{~nm}$ Au NPs. To remove the excess of reagents, the colloids were centrifuged at $1520 \mathrm{~g}$ for $30 \mathrm{~min}$ and redispersed in the same volume of water.

LbL Fabrication of Au NP Thin Films. LbL Au NPs substrates were obtained following a method previously reported by Vial et al. ${ }^{37}$ In brief, glass slides were activated with piranha solution $\left(\mathrm{H}_{2} \mathrm{SO}_{4} /\right.$ $\mathrm{H}_{2} \mathrm{O}_{2}$ 70:30) for $30 \mathrm{~min}$ and then copiously rinsed with water and stored in water until use. Glass slides were cut to dimensions of $5 \times$ $20 \mathrm{~mm}$. The glass slides were rinsed with water again and dried under an air stream.

The activated glass slides were immersed in Eppendorf tubes containing an aqueous solution of $1 \mathrm{mg} \mathrm{mL}^{-1}$ PDDA $(0.5 \mathrm{M} \mathrm{NaCl})$ for $15 \mathrm{~min}$, rinsed with water, and then dried. The process was repeated twice but alternating the polyelectrolyte aqueous solution: PSS solution $\left(1 \mathrm{mg} \mathrm{mL}^{-1}, 0.5 \mathrm{M} \mathrm{NaCl}\right)$ first, and then PDDA solution (1 $\mathrm{mg} \mathrm{mL} \mathrm{m}^{-1}, 0.5 \mathrm{M} \mathrm{NaCl}$ ). Both polyelectrolyte solutions were sonicated for $10 \mathrm{~min}$ before use. The rinsing step with water was performed three-fold by dipping the glass slides in different Eppendorf tubes for $1 \mathrm{~min}$. For the Au NPs deposition, the glass slides were immersed in the previously prepared $0.92 \mathrm{mM}$ Au NPs solution for 4 $\mathrm{h}$ under gentle stirring followed by rinsing with water and drying with an air stream. Then, the substrates were immersed again in the aqueous solution of PDDA $\left(1 \mathrm{mg} \mathrm{mL} L^{-1}, 0.5 \mathrm{M} \mathrm{NaCl}\right)$ for $15 \mathrm{~min}$, rinsed with water, and then dried. Finally, a new immersion of the glass substrates was performed in the Au NPs solution for $4 \mathrm{~h}$, followed by rinsing with water and drying with an air stream. Note that for each immersion, fresh Au NPs or PDDA solutions were employed. Au substrates were kept in Eppendorf tubes until use.

Fabrication of Au@MIP Thin Films for SERS Sensing. First, a prepolymerization mixture was prepared as follows: to $3.95 \mathrm{~mL}$ of DMF (porogen solvent) $69 \mathrm{mg}$ of pyrene (template) or fluoranthene (template) was added. After ultrasound sonication for $3 \mathrm{~min}, 57 \mu \mathrm{L}$ of 
MAA (0.67 mmol), $835 \mu \mathrm{L}$ of DVB (4.69 mmol), and $170 \mu \mathrm{L}$ of AIBN solution $\left(40 \mathrm{mg} \mathrm{mL}^{-1}\right.$ in DMF) were added. In the case of $\mathrm{Au} @$ NIP thin films, the procedure was the same except that no template molecule was added to the prepolymerization mixture. The prepolymerization mixture was purged with nitrogen for $10 \mathrm{~min}$ and introduced in an oven at $60{ }^{\circ} \mathrm{C}$ for a certain time (noted as prepolymerization time). Thus, experiments with prepolymerization time ranging from 1 to $4 \mathrm{~h}$ were performed Then, $50 \mu \mathrm{L}$ of the prepolymerization mixture was deposited on the Au NPs thin film by spin-coating (PRSI 4E, GME Ltd, Surrey, UK) for 20 s. Experiments at different spin-coating rates $(1000,2000$, and $4000 \mathrm{rpm})$ were performed in order to modulate the thickness of the polymer film. After purging with nitrogen for $10 \mathrm{~min}$, the substrate was heated at 60 ${ }^{\circ} \mathrm{C}$ for $30 \mathrm{~min}$ for curing. Then, template removal was performed by washing the resulting Au@MIP platforms twice with DMF at $60{ }^{\circ} \mathrm{C}$ for 1 h. Finally, the Au@MIP substrates were dried with air and kept until use. Au@NIP platforms were subjected to the same DMF washing procedure to ensure the same experimental conditions for both substrates.

SERS Detection of PAHs. For SERS detection of a single analyte or their PAHs mixtures, Au@MIP substrates were immersed in $10 \mathrm{~mL}$ of aqueous PAH solutions (concentrations ranging from $10^{-5}$ to $10^{-10}$ $\mathrm{M})$ and kept for $1 \mathrm{~h}$ at $60^{\circ} \mathrm{C}$. PAH stock solutions $\left(10^{-2} \mathrm{M}\right)$ were prepared in DMF. After incubation, Au@MIP platforms were rinsed with water and air-dried. The procedure was analogous to Au@NIP substrates.

SERS Detection of Pyrene in Real Samples. Two different real samples were selected: creek water and seawater. The creek water was collected from a creek located in the forest that surrounds the Vigo campus. The seawater from the Atlantic ocean was collected at Portomaior Beach in Bueu (Pontevedra). Both samples were filtered with a $0.22 \mu \mathrm{m}$ filter (Whatman) prior to analysis. Pyrene-spiked solutions (concentrations ranging from $10^{-5}$ to $10^{-10} \mathrm{M}$ ) were prepared by using both matrices. The rest of the procedure was analogous as for Au@MIP substrates.

Characterization. UV-visible-NIR absorption spectra were recorded using an Agilent 8453 spectrophotometer. A profilometry equipment Dektak XT (Bruker, Massachusetts, US) of high resolution $(1 \mathrm{~nm})$ was employed to determine the thickness of the films of Au@ MIPs and Au@NIPs. For each sample, three scans (ranging from 2 to $8 \mathrm{~mm}$ ) in different zones were performed. Transmission electron microscopy analysis was performed in a JEOL JEM 1010 microscope operating at an acceleration voltage of $100 \mathrm{kV}$. Scanning electron microscopy (SEM) images were obtained using a JEOL JSM-6700F FEG microscope operating at an acceleration voltage of $10.0 \mathrm{kV}$. Optimized geometries of the different PAHs were calculated using the B3LYP/6-31G* density functional theory and the Gaussian 09 program. SERS measurements were conducted with a Renishaw InVia Reflex system. The spectrograph used a high-resolution grating (1200 grooves $\mathrm{cm}^{-1}$ ) with additional band-pass filter optics, a confocal microscope, and a 2D-CCD camera. Laser excitation was carried out at $785 \mathrm{~nm}, 1.99 \mathrm{~mW}$ of maximum power, and $0.5 \mathrm{~s}$ of acquisition time. SERS images were obtained using a SERS point-mapping method with a $50 \times$ objective (N.A. 0.75 ) or with a $10 \times$ objective (N.A. 0.25). A spectral image was created by measuring the SERS spectrum of each pixel of the image, one at a time. The SERS images of each pixel were decoded using the characteristic peak intensities of each PAH using WiRE software V 4.3 (Renishaw, U.K.).

\section{RESULTS AND DISCUSSION}

Design and Optimization of Au@MIP Thin Films. A hybrid Au@MIP thin film platform based on Au NPs and a MIP was developed following a two-step procedure consisting of (1) the fabrication of a highly SERS-sensitive Au NPs substrate and (2) the subsequent deposition of a uniform MIP coating and removal of the template molecule. The Au NPs provided the platform with plasmonic sensing capabilities, whereas the MIP allowed the selective hosting of a target analyte through molecular recognition.

The plasmonic substrate was fabricated as previously reported $^{38}$ via electrostatic LbL assembly of citrate-stabilized $60 \mathrm{~nm}$ Au nanospheres (Figures S1 and S2). The resulting Au NPs platform featured a plasmon band at $516 \mathrm{~nm}$, ascribed to the dipole resonance of individual Au NPs, as well as a broad band at $850 \mathrm{~nm}$, due to the plasmon coupling between NPs in close proximity (Figure S2B). The Au NP assembly was characterized by SEM showing a uniform NP distribution along the glass surface (Figure S2C).

The MIP thin film deposition over the Au NP assembly was carried out through the copolymerization of MAA with DVB, a cross-linking agent, in the presence of the template molecule (pyrene or fluoranthene) and azobisisobutyronitrile (AIBN) as the initiator followed by template extraction from the crosslinked polymer matrix (see Scheme 1). MAA has been

\section{Scheme 1. Schematic Representation of Au@MIP} Fabrication Process

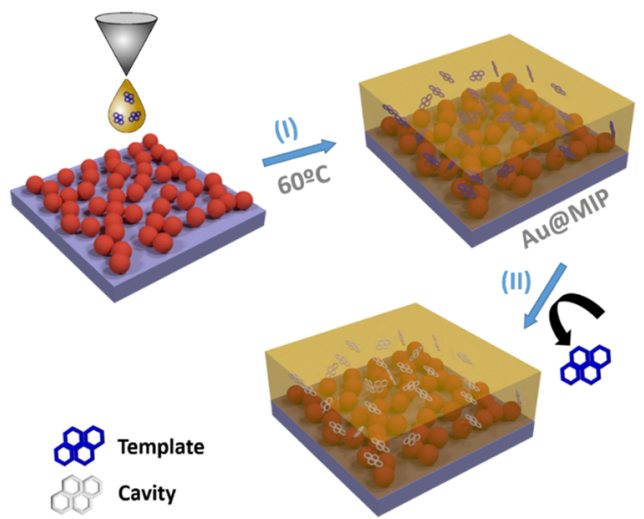

extensively used as a MIP monomer due to its capability to act both as a hydrogen bond and proton donor and as a hydrogen bond acceptor. ${ }^{39}$ Moreover, this monomer has been previously used for pyrene recognition. ${ }^{34,40,41}$ The prepolymerization solution (MAA, DVB, AIBN, and the template molecule) was heated at $60{ }^{\circ} \mathrm{C}$ till reaching the appropriate viscosity and degree of crosslinking and then spin-coated on the Au NPs assembly. ${ }^{42}$ Subsequently, the substrate was heated at $60{ }^{\circ} \mathrm{C}$ for $30 \mathrm{~min}$ in the absence of oxygen to induce the complete polymerization (curing) of the MIP. Finally, the removal of the template was carried out by DMF washing. Au@NIPs were fabricated as reference samples following the same strategy but in the absence of the template molecule.

The optimization of Au@MIP thin films to achieve a highly sensitive and reliable SERS-based PAH sensor required the analysis of different key parameters. It was carried out considering that a good performance was closely related to the MIP films' ability to trap the maximum amount of the target molecules on/close to the Au NPs surface ${ }^{43}$ and therefore the deposition of a very thin, but uniform, MIP film on the plasmonic substrate was desired. With this aim, the effect of prepolymerization time and spin-coating rate in the MIP formation (thickness and homogeneity) were studied by profilometry using pyrene-based Au@MIPs. The film analysis was performed after pyrene removal from MIP matrix. Although, the monomer:crosslinker molar ratio is crucial for defining the final properties of the polymer in terms of porosity and rigidity, it was fixed to $1: 7$ as previously stablished. ${ }^{44}$ Thus, 

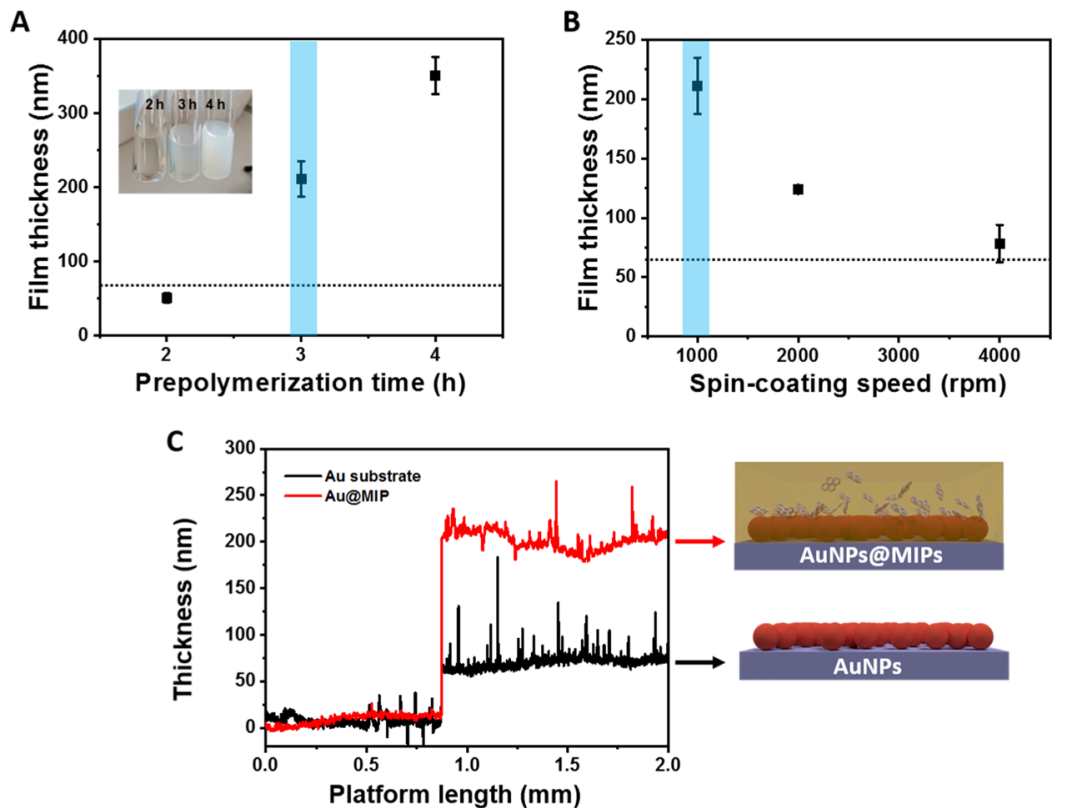

Figure 1. (A,B) Evaluation of the thickness and homogeneity of the MIP film as a function of (A) prepolymerization time and (B) spin-coating rate for Au@MIP hybrid platforms after the removal of pyrene by DMF washing. (C) Profilometry analysis of Au NP substrate (black spectrum) and $\mathrm{Au} @$ MIP prepared under the optimum conditions (red spectrum). The shadow regions in A and B indicate the selected parameters for the Au@ MIP thin film fabrication. All Au@MIPs were fabricated using pyrene as the template molecule.
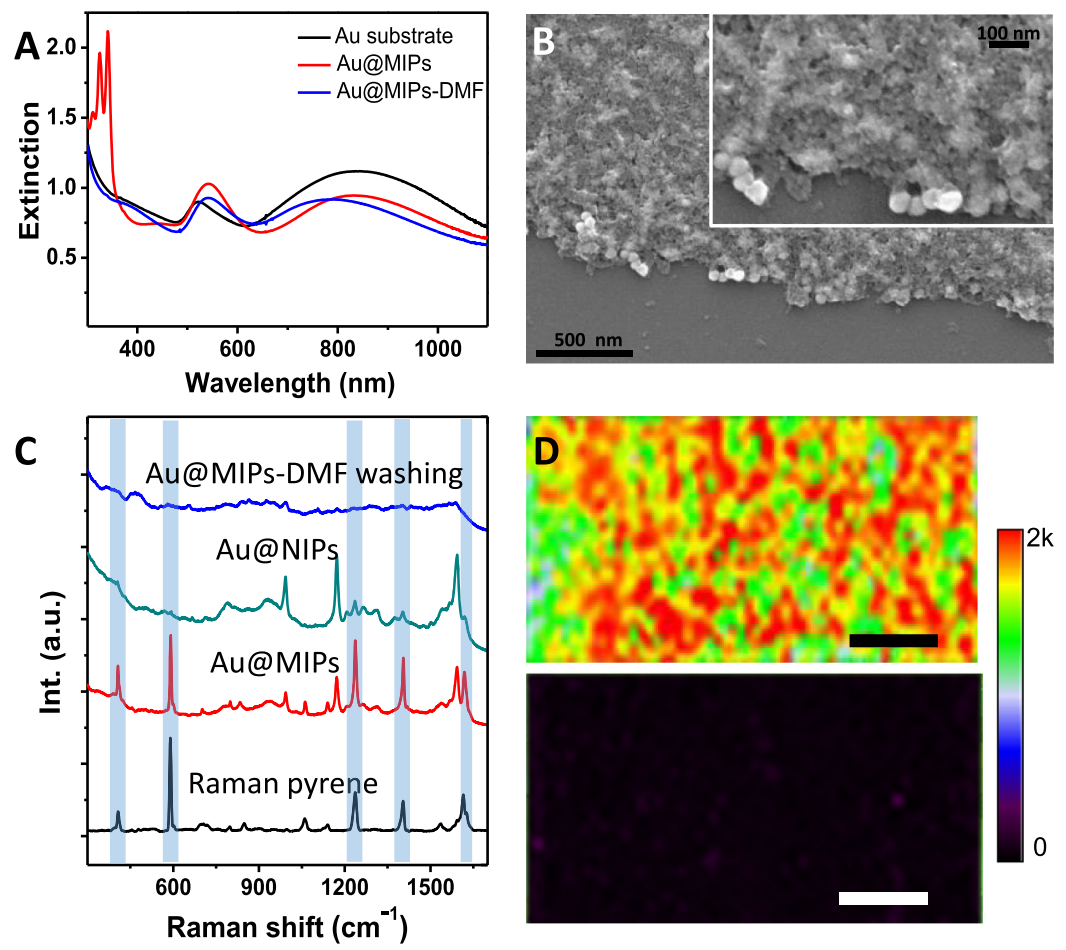

Figure 2. (A) UV-visible-NIR extinction spectra of Au NP assembly (black), Au@MIP before (red) and after (blue) DMF washing. (B) SEM image of Au@MIP. (C) SERS spectra of Au@MIP before (red) and after (blue) DMF washing and Au@NIP before DMF washing (green), and Raman spectrum of pyrene (black). (D) SERS intensity mappings of Au@MIP recorded at $594 \mathrm{~cm}^{-1}$ before (top) and after (bottom) DMF washing. SERS spectra were acquired with laser excitation at $785 \mathrm{~nm}, 10 \times$ objective, $0.5 \mathrm{~s}$ acquisition time, and laser power of $1.99 \mathrm{~mW}$. Scale bars are $1000 \mu \mathrm{m}$. All Au@MIPs are pyrene-based.

prepolymerization time was varied between 0 and $4 \mathrm{~h}$ and the spin-coating rate between 1000 and $4000 \mathrm{rpm}$. Figure 1 summarizes the effect of both parameters in the MIP film thickness. The pristine gold substrate, without MIP thin film, presents an average thickness of $63.3 \pm 1.5 \mathrm{~nm}$ (see Figure
1C), which is in good agreement with Au NP dimensions (ca. $60 \mathrm{~nm}$ ). As shown in Figure 1A, for prepolymerization times of $2 \mathrm{~h}$ or shorter, the measured average thickness was close to $\sim 60 \mathrm{~nm}$ indicating that the MIP film was negligible, most probably due to the low viscosity of the solution which 

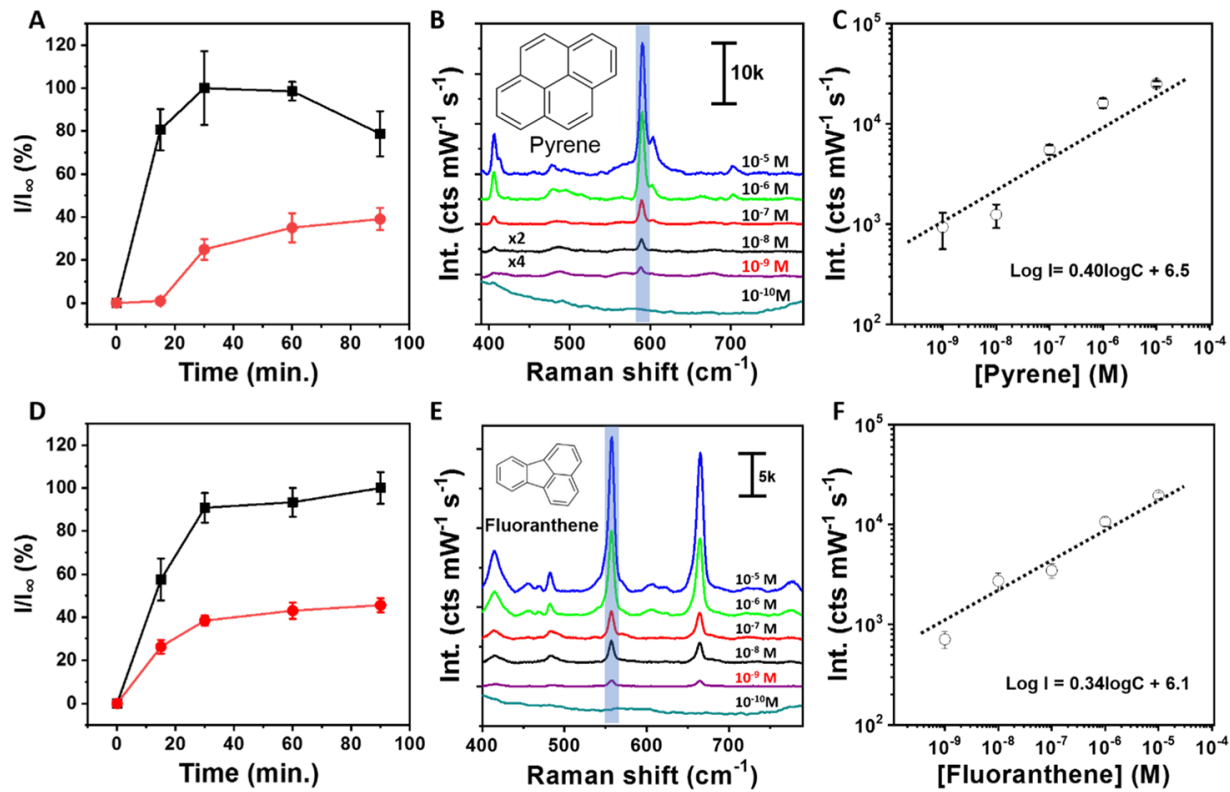

Figure 3. (A,D) Time-dependent SERS intensity changes $\left(I / I_{\infty}(\%)\right)$ recorded at $594 \mathrm{~cm}^{-1}$ upon the pyrene-based Au@MIPs uptake of $10^{-7} \mathrm{M}$ pyrene (A) or at $557 \mathrm{~cm}^{-1}$ upon the fluoranthene-based Au@MIPs uptake of $10^{-7} \mathrm{M}$ fluoranthene (D). Control experiments with Au@NIPs (in red) are also shown. (B,E) SERS spectra of (B) pyrene or (E) fluoranthene recorded from aqueous solutions at different concentrations. The insets show the molecular structure of (B) pyrene or (E) fluoranthene. (C,F) SERS intensity at $594 \mathrm{~cm}^{-1}$ (C) or at $557 \mathrm{~cm}^{-1}(\mathrm{~F})$ as a function of pyrene or fluoranthene concentration, respectively. SERS measurements were performed with a $785 \mathrm{~nm}$ laser line, $0.5 \mathrm{~s}$ acquisition time, and $1.74 \mathrm{~mW}$ maximum power.

hindered film formation by spin-coating. Nevertheless, above 2 h, Au@MIP film thickness increases with the prepolymerization time, which could be ascribed to an increase in the viscosity of the prepolymerization mixture due to entanglement or crosslinking processes. Moreover, it should be noted that above $3 \mathrm{~h}$, the resulting films are rather uniform (with residual standard deviation, RSD, between 7 and 13\%) and thick enough to ensure the complete burial of the Au NPs in the MIP matrix. While $3 \mathrm{~h}$ of prepolymerization gave rise to a homogenous thin film of $211 \pm 23.7 \mathrm{~nm}$, much thicker films were obtained for $4 \mathrm{~h}$ (Figure 1C). As the plasmon-induced enhancement of Raman signals is just expected for molecules located few $\mathrm{nm}$ away from the Au surface, $3 \mathrm{~h}$ prepolymerization time was selected to analyze the spin-coating rate. As shown in Figure 1B, an increase in the rate from 1000 to 4000 rpm decreases the average film thickness from ca. 200 to $80 \mathrm{~nm}$ without affecting the uniformity of the film. ${ }^{44}$ Taking into account that $\mathrm{Au}$ particles are around $60 \mathrm{~nm}, 1000 \mathrm{rpm}$ was selected as the spin-coating rate. Therefore, the experimental conditions for the Au@MIPs fabrication were monomer/ crosslinker ratio of $1: 7,3 \mathrm{~h}$ of prepolymerization time, and $1000 \mathrm{rpm}$ spin-coating rate. A similar analysis was also performed for the fabrication of fluoranthene-based Au@ MIPs and Au@NIPs, obtaining similar results.

Au@MIPs Characterization. Next, the optimized Au@ MIP and Au@NIP thin films were further characterized by UV-visible-NIR spectroscopy, SEM, and SERS spectroscopy. The extinction spectra of Au@MIP or Au@NIP thin films (Figure 2A) show similar optical features to those exhibited by $\mathrm{Au}$ NPs substrates before the MIP deposition. The slight differences observed in the main peak position are most probably due to the minor changes in the Au NP distribution and in the local refractive index produced by the MIP coating. UV-visible-NIR spectroscopy was also useful to monitor the removal of the template from the resulting MIP matrix. As shown in Figure 2A, before the DMF washing, the pyrenebased Au@MIP presents two intense absorption bands between 300 and $360 \mathrm{~nm}$, which can be assigned to the electronic excitation of pyrene, ${ }^{45}$ indicating molecule incorporation in the polymer matrix. Nevertheless, those bands completely disappeared after two cycles of Au@MIP immersion $(1 \mathrm{~h})$ in $\mathrm{DMF}$ at $60{ }^{\circ} \mathrm{C}$, confirming the removal of pyrene from the MIP matrix (blue spectrum in Figure $2 \mathrm{~A}$ ). In the case of fluoranthene-based Au@MIPs, it was performed by monitoring the characteristic absorption bands from fluoranthene at 260 and $380 \mathrm{~nm}$ (data not shown). On the other hand, SEM characterization (Figure 2B) reveals a uniform $\mathrm{Au}$ NP assembly monolayer covered with a homogenous thin polymer film. Additionally, Au@MIPs and $\mathrm{Au} @$ NIPs were characterized by SERS before and after the template removal. As shown in Figure 2C, the SERS spectrum of the pyrene-based Au@MIPs before DMF washing is dominated by the characteristic signals of pyrene: ring $\mathrm{C}=\mathrm{C}$ stretching (1407 and $1242 \mathrm{~cm}^{-1}$ ), ring deformation (409 $\left.\mathrm{cm}^{-1}\right)$, ring breathing $\left(594 \mathrm{~cm}^{-1}\right)$, ring $\mathrm{C}=\mathrm{C}$ stretching $(1594$ and $1628 \mathrm{~cm}^{-1}$ ), and $\mathrm{CH}$ bending $\left(1067\right.$ and $\left.1143 \mathrm{~cm}^{-1}\right){ }^{46}$ Furthermore, other SERS signals not attributed to pyrene but to the polymer matrix are observed. The fact that those signals also appear in the SERS spectrum of the Au@NIP before DMF washing (green spectrum in Figure 2C) may indicate that they correspond to the polymer reactants in excess. Interestingly, after the DMF washing step, none of the characteristic SERS peaks from pyrene or other reactants were observed (blue spectrum in Figure 2C), indicating their complete removal from the Au@MIP thin film. Finally, the uniformity of the Au@MIP thin films was investigated by recording SERS intensity mappings at $594 \mathrm{~cm}^{-1}$ (ring breathing peak of pyrene) over extended film areas (typically $5200 \times 3000 \mu \mathrm{m}$ with $100 \mu \mathrm{m}$ step size) (Figure 2D). Before DMF washing, the SERS mappings showed a highly intense and homogeneous 
pyrene signal over the Au@MIP thin films (RSD between 10 and $15 \%$ for the SERS intensity) (Figure 2D). Nevertheless, the signal completely disappeared over the whole thin film (Figure 2D) after the washing step, confirming its complete removal. Analogous results were obtained for fluoranthenebasedAu@MIPs.

SERS Performance of Au@MIPs. So far, we demonstrated that a new hybrid platform could be fabricated using as building blocks Au NP assemblies and pyrene- or fluoranthenebased MIP thin films. Next, the sensing capabilities of both types of Au@MIPs were evaluated.

First, we investigated the pyrene or fluoranthene uptake kinetics by its corresponding Au@MIP using SERS. Despite its low affinity for the gold surface, the PAH should be selectively and quickly trapped by the $3 \mathrm{D}$ cavities created during the MIP fabrication process. The amount of adsorbed PAH is proportional to the corresponding SERS peak intensity. Thus, Au@MIP and Au@NIP substrates were incubated at $60{ }^{\circ} \mathrm{C}$ over $90 \mathrm{~min}$ in $10^{-7} \mathrm{M}$ pyrene or fluoranthene. The time-dependent uptake was studied at 594 or $557 \mathrm{~cm}^{-1}$ for pyrene or fluoranthene, respectively. As shown in Figure 3A,D the pyrene or fluoranthene uptake rate was much superior for Au@MIP than for Au@NIP substrates. Thus, the maximum uptake (equilibrium amount) was achieved after $30 \mathrm{~min}$ for pyrene and $60 \mathrm{~min}$ for fluoranthene in the case of Au@MIPs. Nevertheless, Au@NIPs did not reach values larger than 40\% of the equilibrium amount for both targets even after $90 \mathrm{~min}$ of incubation. The experiment was also carried out for $10^{-8} \mathrm{M}$ pyrene (Figure S3), observing slightly slower uptake kinetics for Au@MIPs (equilibrium time around $60 \mathrm{~min}$ ) and no pyrene detection for Au@NIPs.

We concluded that $60 \mathrm{~min}$ was the optimal time of incubation for generating a SERS signal corresponding to about $100 \%$ of the saturation value, independently of the template. These results clearly support the potential applicability of our plasmonic chemical sensor.

Second, the sensing capabilities were analyzed through the determination of the limit of detection (LOD) and quantitative SERS detection region for pyrene and fluoranthene in aqueous media using pyrene- and fluoranthene-based Au@MIPs, respectively. Thus Au@MIPs thin films were immersed for 1 $\mathrm{h}$ at $60{ }^{\circ} \mathrm{C}$ in aqueous solutions of pyrene or fluoranthene at concentrations ranging from $10^{-5}$ to $10^{-10} \mathrm{M}$ and then analyzed by SERS. Figure $3 \mathrm{~B}, \mathrm{E}$ shows representative SERS spectra acquired for the Au@MIPs after incubation with the different PAH concentrations. The characteristic SERS peaks of pyrene (Figure 3B) and fluoranthene (Figure 3E) decreased in intensity with the concentration being distinguishable up to molecule concentrations of $10^{-9} \mathrm{M}$. A detailed vibrational band assignment of both PAHs can be found in Table S1. Besides, it was found that within a certain concentration range $\left(10^{-5}\right.$ to $10^{-8} \mathrm{M}$ for pyrene and $10^{-5}$ to $10^{-9} \mathrm{M}$ for fluoranthene), the SERS response was homogeneous (with RSD $\leq 10 \%$ ) along the whole Au@MIP, indicating its uniform spatial distribution as well as the good quality of the platform. It is also evidenced in the SERS mapping acquired for $10^{-5} \mathrm{M}$ pyrene (Figure S4A). Whereas, lower PAH concentrations led to RSD $\geq 10 \%$ in the SERS signal along the Au@MIP thin film. Therefore, systematic SERS mapping measurements were necessary in order to determine the LOD with accuracy. Thus, the experimental data corresponding to $10^{-9} \mathrm{M}$ in Figure $3 \mathrm{C}$ is an average of 441 points. This behavior is clearly reflected in the SERS mapping recorded for $10^{-9} \mathrm{M}$ pyrene (Figure S4B).
Importantly, the LODs reached by the Au@MIP thin films fabricated for pyrene or fluoranthene detection are lower than those established by the regulation of the European Union (8.0 $\times 10^{-9}$ to $\left.4.0 \times 10^{-8} \mathrm{M}\right)$ in different types of foods. ${ }^{47} \mathrm{We}$ strongly believe that this imprinting strategy could be easily extended for the ultrasensitive detection of other PAHs.

The sensitivity and efficiency of the Au@MIP were also demonstrated by comparing its LOD for pyrene and fluoranthene with that for Au@NIPs (Figures S5 and S6) and pristine Au substrate. SERS signals obtained for Au@NIPs and $\mathrm{Au}$ NPs substrates were the result of unspecific molecule adsorptions to the hydrophobic polymeric matrix (NIP) and to the metal surface. Overall, Au@MIP improved the LOD by 2 and 3 orders of magnitude with respect to Au@NIPs and pristine Au substrate, respectively.

The PAH binding to the cavities of the Au@MIP can be modeled by employing the Langmuir-Freundlich (LF) isotherm or just the Freundlich isotherm at low concentration ranges. ${ }^{48}$ Considering the working concentration range for pyrene and fluoranthene, we described the Au@MIPs behavior through the Freundlich model

$$
\theta \propto I=K_{\mathrm{a}} \cdot C^{\mathrm{m}}
$$

where $\theta$ is the amount of pyrene complexed in the Au@MIP, $I$ the SERS intensity (in counts $\mathrm{mW}^{-1} \mathrm{~s}^{-1}$ ), $K_{\mathrm{a}}$ the binding constant, $C$ the pyrene molar concentration, and $m$ the heterogeneity index. $m$ varies between 0 (complete heterogeneous material) and 1 (homogeneous material). The previous equation can be linearized as follows

$$
\log I=\log \left(K_{\mathrm{a}}\right)+m \log C
$$

Figure 3C,F shows the SERS intensity versus concentration (both at log scale) for pyrene $\left(594 \mathrm{~cm}^{-1}\right)$ and fluoranthene $\left(557 \mathrm{~cm}^{-1}\right)$, respectively. Each experimental point corresponds to at least 20 random points recorded and averaged for each concentration on the Au@MIP thin film. Moreover, the reported average values correspond to two different hybrid platforms evaluated for each concentration, demonstrating the high reproducibility between sensors. The data revealed a linear variation of the SERS signal with the pyrene (Figure 3C) and fluoranthene (Figure 3D) concentration from $10^{-5}$ to $10^{-9}$ M. It could be fitted to eq 2 obtaining the empirical formula: $\log I=6.5+0.40 \log C\left(R^{2}=0.96\right)$ for pyrene-based Au@MIP and $\log I=6.10+0.34 \log C\left(R^{2}=0.99\right)$ for fluoranthenebased Au@MIP. Importantly, it allowed to obtain the binding constant between PAH and Au@MIPs being $3.2 \times 10^{6}$ and 1.3 $\times 10^{6} \mathrm{M}^{-1}$ for pyrene and fluoranthene, respectively. The same analysis performed for fluoranthene but using the SERS intensity data at $665 \mathrm{~cm}^{-1}$ instead of at $557 \mathrm{~cm}^{-1}$ (Figure S7) revealed similar behavior and binding constant.

To evaluate the specific recognition ability of pyrene- and fluoranthene-based Au@MIPs, a SERS analysis was performed by using mixtures of three different PAHs namely; pyrene, fluoranthene, and benzo[a]pyrene. Benzo[a]pyrene was selected because it has a larger size than pyrene and fluoranthene. According to the calculated optimized geometry (see experimental part and Figure S8), the dimensions of these three PAHs decrease as follows: benzo[a]pyrene $(9.820 \AA)$ > fluoranthene $(9.467 \AA)>$ pyrene $(9.013 \AA)$. As shown in Figure $4 \mathrm{~A}$ the spectral region between 300 and $700 \mathrm{~cm}^{-1}$ is sufficient to clearly identify representative bands corresponding to each PAH. Long-range representative SERS spectra of each 


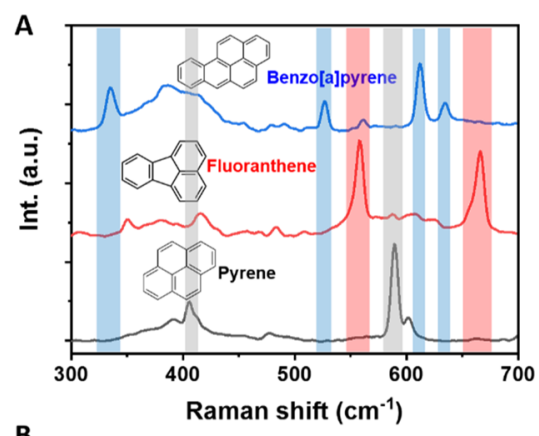

B

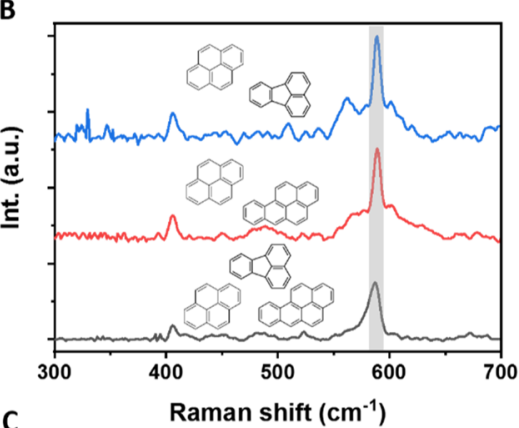

C

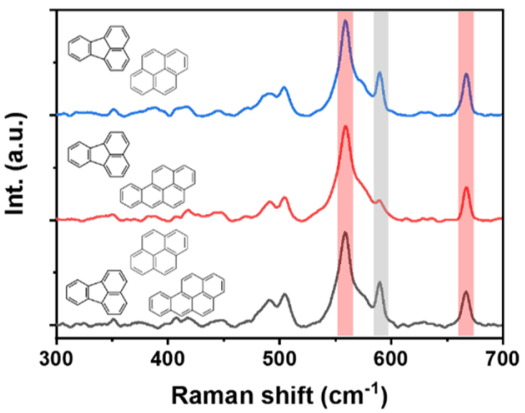

Figure 4. (A) Representative SERS spectra of benzo[a]pyrene (blue), fluoranthene (red), and pyrene (black). (B) SERS spectra recorded using pyrene-based Au@MIPs of different mixtures: pyrene/ fluoranthene (blue), pyrene/benzo[a]pyrene (red), and pyrene/ fluoranthene/benzo[a]pyrene (black). (C) SERS spectra recorded using fluoranthene-based Au@MIPs of the following aqueous mixtures: fluoranthene/pyrene (blue), fluoranthene/benzo[a]pyrene (red), and pyrene/fluoranthene/benzo[a]pyrene (black). The concentration of the three PAHs was $10^{-8} \mathrm{M}$ in all mixtures.

PAH have been included in Figure S9 and their vibrational band assignment in Table S1.

First, the selectivity of pyrene-based Au@MIP was evaluated through SERS analysis after its incubation in three different aqueous mixtures of PAHs (pyrene/fluoranthene, pyrene/ benzo $[a]$ pyrene, and pyrene/fluoranthene/benzo[a]pyrene). In all cases, the SERS spectra of the Au@MIPs (Figure 4B) just showed the characteristic signals of pyrene. The fact that no signals from fluoranthene and bezo[a]pyrene were evidenced could indicate cavity-size selectivity as the pyrene template is the smallest PAH used. Thus, in the present case, a good selectivity (with a LOD of $1 \mathrm{nM}$ ) for pyrene was obtained.

Similar experiments were performed for fluoranthene-based $\mathrm{Au} @$ MIPs using the following PAHs aqueous mixtures: fluoranthene/pyrene, fluoranthene/benzo $[a]$ pyrene, and fluoranthene/benzo $[a]$ pyrene/pyrene. In this case, the SERS analysis of the Au@MIPs (Figure 4C) showed the signatures of fluoranthene as well as pyrene but none from benzo[a]pyrene. This behavior could be also explained in terms of cavity-size selectivity because the molecular dimensions of fluoranthene are larger than those of pyrene but smaller than those for benzo $[a]$ pyrene. These results demonstrated that the suitable choice of the template PAH modified the selectivity of Au@MIPs.

Ultrasensitive SERS-Based Detection of Pyrene in Real Samples. In order to demonstrate the practical applicability of our developed plasmonic sensor, pyrene was chosen as the target $\mathrm{PAH}$ due to the large selectivity of the pyrene-based Au@MIPs. This PAH is included in the United States Environmental Protection Agency (US EPA) priority $\mathrm{PAH}$ list. Thus, pyrene detection was accomplished using pyrene-based Au@MIPs in two real matrices: creek water (Figure 5) and seawater (Figure S10). Both kinds of real
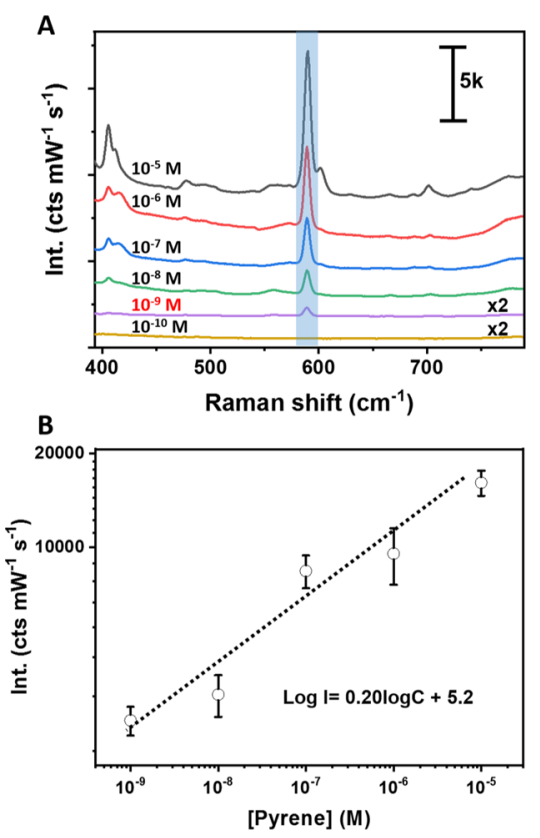

Figure 5. (A) SERS spectra of pyrene obtained from spiked creek water solutions at different concentrations using as sensing platform pyrene-based Au@MIPs. (B) SERS intensity at $594 \mathrm{~cm}^{-1}$ as a function of pyrene concentration.

samples were collected and filtered prior to use, then pyrene was directly spiked at different concentrations ranging from $10^{-5}$ to $10^{-10} \mathrm{M}$. All experimental procedures were performed as previously described. Figures 5A and S10A show representative SERS spectra acquired for the different pyrene concentrations. Remarkably, the characteristic SERS peaks of pyrene were again distinguished up to molecular concentrations of $10^{-9} \mathrm{M}$, independently of the matrix sample. The analysis of the average SERS intensity at $594 \mathrm{~cm}^{-1}$ as a function of pyrene concentration revealed a quantification region in the whole range (Figures $5 \mathrm{~B}$ and $\mathrm{S} 10 \mathrm{~B}$ ), which can be expressed by the empirical formulas: $\log I=5.20+0.20 \log$ $C\left(R^{2}=0.97\right)$ in creek water and $\log I=6.10+0.37 \log C\left(R^{2}\right.$ $=0.95)$ in seawater. Additionally, the binding constants, $K_{a}$ were calculated as being $1.6 \times 10^{5}$ and $1.3 \times 10^{6} \mathrm{M}^{-1}$ for seawater and creek water, respectively. In the case of seawater, $K_{\mathrm{a}}$ was similar to that previously obtained in Milli-Q water, suggesting no matrix interference. Whereas $K_{\mathrm{a}}$ in creek water was 1 order of magnitude lower than that in Milli-Q water which could be translated into a matrix effect. Nevertheless, 
this matrix effect seemed to have no influence on the sensitivity of the plasmonic sensor.

\section{CONCLUSIONS}

In summary, we developed a reliable and sensitive SERS-based hybrid sensor consisting of a Au NP assembly covered by a uniform MIP thin film. In order to achieve homogeneous MIP coatings, parameters such as prepolymerization time and spincoating rate were finely modulated. The combination of $\mathrm{Au}$ NPs and MIPs hence solved the main drawbacks of each component when used separately. Analysis of the SERS performance of the Au@MIP thin films fabricated using pyrene or fluoranthene as the template revealed that: (i) the presence of MIPs allowed detecting molecules without an affinity for gold, such as low molecular-weight PAHs; (ii) the pyrene- and fluoranthene-based sensing platforms showed limits of detection for pyrene and fluoranthene, respectively, up to 100-fold times lower than those achieved with the corresponding Au@NIP thin films; (iii) for both analyzed PAHs, a quantification region is observed and their binding constants can be calculated; (iv) the template molecule used during the Au@MIPs fabrication may improve the selectivity for each $\mathrm{PAH}$; and (v) the plasmonic sensor might be employed to analyze pyrene in real samples (creek water and seawater) without affecting its sensitivity as a result of a matrix effect.

\section{ASSOCIATED CONTENT}

\section{(s) Supporting Information}

The Supporting Information is available free of charge at https://pubs.acs.org/doi/10.1021/acssensors.9b01882.

Characterization of Au NPs, additional SERS characterization mappings, and Raman assignments (PDF)

\section{AUTHOR INFORMATION}

\section{Corresponding Authors}

Jorge Pérez-Juste - Centro Singular de Investigaciones Biomédicas (CINBIO) y Departamento de Qúmica Física, Universidade de Vigo, 36310 Vigo, Spain; @ orcid.org/00000002-4614-1699; Email: juste@uvigo.es

Isabel Pastoriza-Santos - Centro Singular de Investigaciones Biomédicas (CINBIO) y Departamento de Química Física, Universidade de Vigo, 36310 Vigo, Spain; (1) orcid.org/00000002-1091-1364; Email: pastoriza@uvigo.es

\section{Authors}

Alexander Castro-Grijalba - Centro Singular de Investigaciones Biomédicas (CINBIO) y Departamento de Química Física, Universidade de Vigo, 36310 Vigo, Spain; INFIQC, Centro Láser de Ciencias Moleculares, Departamento de Fisicoquímica, Facultad de Ciencias Químicas, Universidad Nacional de Córdoba, 5000 Córdoba, Argentina

Verónica Montes-García - Centro Singular de Investigaciones Biomédicas (CINBIO) y Departamento de Química Física, Universidade de Vigo, 36310 Vigo, Spain; 이이.org/00000002-3235-0545

María José Cordero-Ferradás - Centro Singular de Investigaciones Biomédicas (CINBIO) y Departamento de Química Física, Universidade de Vigo, 36310 Vigo, Spain

Eduardo Coronado - INFIQC, Centro Láser de Ciencias Moleculares, Departamento de Fisicoquímica, Facultad de
Ciencias Químicas, Universidad Nacional de Córdoba, 5000 Córdoba, Argentina; 이이이.org/0000-0002-0748-6803

Complete contact information is available at:

https://pubs.acs.org/10.1021/acssensors.9b01882

\section{Author Contributions}

A.C.-G and V.M.-G. contributed equally to this work. The manuscript was written through the contributions of all authors. All authors have given approval to the final version of the manuscript.

\section{Notes}

The authors declare no competing financial interest.

\section{ACKNOWLEDGMENTS}

This work was supported by the Ministerio de Economía y Competitividad (MINECO, Spain) under the Grant MAT2016-77809-R, Xunta de Galicia/FEDER under the Grant GRC ED431C 2016-048, Fundación Ramón Areces (SERSforSafety), IBEROS (0245_IBEROS_1_E) and FOODSENS cofunded by FEDER through the program Interreg $\mathrm{V}-\mathrm{A}$ España-Portugal (POCTEP) 2014-2020 and the European Union (European Regional Development Fund-ERDF). A.C.G. acknowledges CONICET postdoctoral scholarship from the Argentinean government. The authors also thank CACTI (Center for Scientific and Technological support) at the University of Vigo for its valuable support in profilometry analysis and Daniel García-Lojo for its valuable support in SERS analysis.

\section{REFERENCES}

(1) Chen, L.; Wang, X.; Lu, W.; Wu, X.; Li, J. Molecular imprinting: perspectives and applications. Chem. Soc. Rev. 2016, 45, 2137-2211.

(2) Speltini, A.; Scalabrini, A.; Maraschi, F.; Sturini, M.; Profumo, A. Newest applications of molecularly imprinted polymers for extraction of contaminants from environmental and food matrices: A review. Anal. Chim. Acta 2017, 974, 1-26.

(3) Lakshmi, D.; Bossi, A.; Whitcombe, M. J.; Chianella, I.; Fowler, S. A.; Subrahmanyam, S.; Piletska, E. V.; Piletsky, S. A. Electrochemical Sensor for Catechol and Dopamine Based on a Catalytic Molecularly Imprinted Polymer-Conducting Polymer Hybrid Recognition Element. Anal. Chem. 2009, 81, 3576-3584.

(4) Liang, R.-N.; Song, D.-A.; Zhang, R.-M.; Qin, W. Potentiometric Sensing of Neutral Species Based on a Uniform-Sized Molecularly Imprinted Polymer as a Receptor. Angew. Chem., Int. Ed. 2010, 49, $2556-2559$.

(5) Uzun, L.; Turner, A. P. F. Molecularly-imprinted polymer sensors: realising their potential. Biosens. Bioelectron. 2016, 76, 131144.

(6) Chantada-Vázquez, M. P.; Sánchez-González, J.; Peña-Vázquez, E.; Tabernero, M. J.; Bermejo, A. M.; Bermejo-Barrera, P.; MoredaPiñeiro, A. Simple and sensitive molecularly imprinted polymer-Mndoped $\mathrm{ZnS}$ quantum dots based fluorescence probe for cocaine and metabolites determination in urine. Anal. Chem. 2016, 88, 27342741.

(7) Canfarotta, F.; Cecchini, A.; Piletsky, S. Molecularly Imprinted Polymers for Analytical Chemistry Applications; Royal Society of Chemistry, 2018; pp 1-27.

(8) Zaidi, S. A. Latest trends in molecular imprinted polymer based drug delivery systems. RSC Adv. 2016, 6, 88807-88819.

(9) Mirata, F.; Resmini, M. In Molecularly Imprinted Polymers in Biotechnology; Mattiasson, B., Ye, L., Eds.; Springer International Publishing: Cham, 2015; pp 107-129.

(10) Chen, L.; Xu, S.; Li, J. Recent advances in molecular imprinting technology: current status, challenges and highlighted applications. Chem. Soc. Rev. 2011, 40, 2922-2942. 
(11) Ding, S.-Y.; Yi, J.; Li, J.-F.; Ren, B.; Wu, D.-Y.; Panneerselvam, R.; Tian, Z.-Q. Nanostructure-based plasmon-enhanced Raman spectroscopy for surface analysis of materials. Nat. Rev. Mater. 2016, $1,16021$.

(12) Pastoriza-Santos, I.; Kinnear, C.; Pérez-Juste, J.; Mulvaney, P.; Liz-Marzán, L. M. Plasmonic polymer nanocomposites. Nat. Rev. Mater. 2018, 3, 375-391.

(13) Schlücker, S. Surface-Enhanced Raman spectroscopy: Concepts and chemical applications. Angew. Chem., Int. Ed. 2014, 53, 47564795.

(14) Montes-García, V.; Gómez-González, B.; Martínez-Solís, D.; Taboada, J. M.; Jiménez-Otero, N.; de Uña-Álvarez, J.; Obelleiro, F.; García-Río, L.; Pérez-Juste, J.; Pastoriza-Santos, I. Pillar[5]areneBased Supramolecular Plasmonic Thin Films for Label-Free, Quantitative and Multiplex SERS Detection. ACS Appl. Mater. Interfaces 2017, 9, 26372-26382.

(15) Liu, C.; Zhang, X.; Li, L.; Cui, J.; Shi, Y.-e.; Wang, L.; Zhan, J. Silver nanoparticle aggregates on metal fibers for solid phase microextraction-surface enhanced Raman spectroscopy detection of polycyclic aromatic hydrocarbons. Analyst 2015, 140, 4668-4675.

(16) Dribek, M.; Rinnert, E.; Colas, F.; Crassous, M.-P.; Thioune, N.; David, C.; De La Chapelle, M.; Compère, C. Organometallic nanoprobe to enhance optical response on the polycyclic aromatic hydrocarbon benzo [a] pyrene immunoassay using SERS technology. Environ. Sci. Pollut. Res. Int. 2017, 24, 27070-27076.

(17) Ahmad, R.; Griffete, N.; Lamouri, A.; Felidj, N.; Chehimi, M. M.; Mangeney, C. Nanocomposites of gold nanoparticles@ molecularly imprinted polymers: chemistry, processing, and applications in sensors. Chem. Mater. 2015, 27, 5464-5478.

(18) Altintas, Z.; Guerreiro, A.; Piletsky, S. A.; Tothill, I. E. NanoMIP based optical sensor for pharmaceuticals monitoring. Sens. Actuators, B 2015, 213, 305-313.

(19) Gam-Derouich, S.; Jouini, M.; Ben Hassen-Chehimi, D.; Chehimi, M. M. Aryl diazonium salt surface chemistry and graft photopolymerization for the preparation of molecularly imprinted polymer biomimetic sensor layers. Electrochim. Acta 2012, 73, 45-52.

(20) Kong, L.-J.; Pan, M.-F.; Fang, G.-Z.; He, X.-l.; Yang, Y.-k.; Dai, J.; Wang, S. Molecularly imprinted quartz crystal microbalance sensor based on poly (o-aminothiophenol) membrane and Au nanoparticles for ractopamine determination. Biosens. Bioelectron. 2014, 51, 286292.

(21) Wang, Z.; Li, H.; Wang, X.; Jiang, J.; Sun, H.; Wang, L. Determination of 2, 6-Dichlorophenol by Surface-Enhanced Raman Scattering with Molecular Imprinting. Anal. Lett. 2018, 51, 20622072.

(22) Gao, F.; Feng, S.; Chen, Z.; Li-Chan, E. C. Y.; Grant, E.; Lu, X. Detection and quantification of chloramphenicol in milk and honey using molecularly imprinted polymers: Canadian penny-based SERS nano-biosensor. J. Food Sci. 2014, 79, N2542-N2549.

(23) Lv, Y.; Qin, Y.; Svec, F.; Tan, T. Molecularly imprinted plasmonic nanosensor for selective SERS detection of protein biomarkers. Biosens. Bioelectron. 2016, 80, 433-441.

(24) Holthoff, E. L.; Stratis-Cullum, D. N.; Hankus, M. E. A Nanosensor for TNT Detection Based on Molecularly Imprinted Polymers and Surface Enhanced Raman Scattering. Sensors 2011, 11, 2700-2714

(25) Feng, S.; Gao, F.; Chen, Z.; Grant, E.; Kitts, D. D.; Wang, S.; $\mathrm{Lu}, \mathrm{X}$. Determination of $\alpha$-tocopherol in vegetable oils using a molecularly imprinted polymers-surface-enhanced Raman spectroscopic biosensor. J. Agric. Food Chem. 2013, 61, 10467-10475.

(26) Hu, Y.; Feng, S.; Gao, F.; Li-Chan, E. C. Y.; Grant, E.; Lu, X. Detection of melamine in milk using molecularly imprinted polymers-surface enhanced Raman spectroscopy. Food Chem. 2015, $176,123-129$.

(27) Carrasco, S.; Benito-Peña, E.; Navarro-Villoslada, F.; Langer, J.; Sanz-Ortiz, M. N.; Reguera, J.; Liz-Marzán, L. M.; Moreno-Bondi, M. C. Multibranched Gold-Mesoporous Silica Nanoparticles Coated with a Molecularly Imprinted Polymer for Label-Free Antibiotic
Surface-Enhanced Raman Scattering Analysis. Chem. Mater. 2016, 28, $7947-7954$.

(28) Murray, C. F. Polycyclic Aromatic Hydrocarbons (Harvey, Ronald G.). J. Chem. Educ. 1998, 75, 1392.

(29) Gao, P.; da Silva, E. B.; Townsend, T.; Liu, X.; Ma, L. Q. Emerging PAHs in urban soils: Concentrations, bioaccessibility, and spatial distribution. Sci. Total Environ. 2019, 670, 800-805.

(30) Chen, C.-H. S.; Kuo, T.-C.; Kuo, H.-C.; Tseng, Y. J.; Kuo, C.H.; Yuan, T.-H.; Chan, C.-C. Metabolomics of Children and Adolescents Exposed to Industrial Carcinogenic Pollutants. Environ. Sci. Technol. 2019, 53, 5454-5465.

(31) Abdel-Shafy, H. I.; Mansour, M. S. M. A review on polycyclic aromatic hydrocarbons: source, environmental impact, effect on human health and remediation. Egypt. J. Pet. 2016, 25, 107-123.

(32) Dat, N.-D.; Chang, M. B. Review on characteristics of PAHs in atmosphere, anthropogenic sources and control technologies. Sci. Total Environ. 2017, 609, 682-693.

(33) Bansal, V.; Kumar, P.; Kwon, E. E.; Kim, K.-H. Review of the quantification techniques for polycyclic aromatic hydrocarbons (PAHs) in food products. Crit. Rev. Food Sci. Nutr. 2017, 57, 3297-3312.

(34) Villar-Navarro, M.; Martín-Valero, M. J.; Fernández-Torres, R. M.; Callejón-Mochón, M.; Bello-López, M. Á. Easy, fast and environmental friendly method for the simultaneous extraction of the 16 EPA PAHs using magnetic molecular imprinted polymers (mag-MIPs). J. Chromatogr. B: Anal. Technol. Biomed. Life Sci. 2017, 1044-1045, 63-69.

(35) Ncube, S.; Kunene, P.; Tavengwa, N. T.; Tutu, H.; Richards, H.; Cukrowska, E.; Chimuka, L. Synthesis and characterization of a molecularly imprinted polymer for the isolation of the 16 US-EPA priority polycyclic aromatic hydrocarbons (PAHs) in solution. J. Environ. Manage. 2017, 199, 192-200.

(36) Bastús, N. G.; Comenge, J.; Puntes, V. Kinetically controlled seeded growth synthesis of citrate-stabilized gold nanoparticles of up to $200 \mathrm{~nm}$ : size focusing versus Ostwald ripening. Langmuir 2011, 27, 11098-11105.

(37) Vial, S.; Pastoriza-Santos, I.; Pérez-Juste, J.; Liz-Marzán, L. M. Plasmon coupling in layer-by-layer assembled gold nanorod films. Langmuir 2007, 23, 4606-4611.

(38) Bodelón, G.; Montes-García, V.; Costas, C.; Pérez-Juste, I.; Pérez-Juste, J.; Pastoriza-Santos, I.; Liz-Marzán, L. M. Imaging Bacterial Interspecies Chemical Interactions by Surface-Enhanced Raman Scattering. ACS Nano 2017, 11, 4631-4640.

(39) Vasapollo, G.; Sole, R. D.; Mergola, L.; Lazzoi, M. R.; Scardino, A.; Scorrano, S.; Mele, G. Molecularly Imprinted Polymers: Present and Future Prospective. Int. J. Mol. Sci. 2011, 12, 5908-5945.

(40) Lai, J.-P.; Niessner, R.; Knopp, D. Benzo [a] pyrene imprinted polymers: synthesis, characterization and SPE application in water and coffee samples. Anal. Chim. Acta 2004, 522, 137-144.

(41) Song, X.; Li, J.; Xu, S.; Ying, R.; Ma, J.; Liao, C.; Liu, D.; Yu, J.; Chen, L. Determination of 16 polycyclic aromatic hydrocarbons in seawater using molecularly imprinted solid-phase extraction coupled with gas chromatography-mass spectrometry. Talanta 2012, 99, 7582.

(42) Schmidt, R. H.; Haupt, K. Molecularly imprinted polymer films with binding properties enhanced by the reaction-induced phase separation of a sacrificial polymeric porogen. Chem. Mater. 2005, 17, $1007-1016$

(43) Stiles, P. L.; Dieringer, J. A.; Shah, N. C.; Van Duyne, R. P. Surface-enhanced Raman spectroscopy. Annu. Rev. Anal. Chem. 2008, 1, 601-626.

(44) Ginzburg-Turgeman, R.; Mandler, D. Nanometric thin polymeric films based on molecularly imprinted technology: towards electrochemical sensing applications. Phys. Chem. Chem. Phys. 2010, 12, 11041-11050.

(45) Zhu, G.; Zhang, L.; Wang, Y.; Xu, X.; Peng, X. Interactions between pyrene and pyridinium ionic liquids studied by ultravioletvisible spectroscopy. J. Mol. Liq. 2016, 213, 289-293. 
(46) Montes-García, V.; Fernández-López, C.; Gómez, B.; PérezJuste, I.; García-Río, L.; Liz-Marzán, L. M.; Pérez-Juste, J.; PastorizaSantos, I. Pillar[5]arene-Mediated Synthesis of Gold Nanoparticles: Size Control and Sensing Capabilities. Chem.-Eur. J. 2014, 20, 8404-8409.

(47) Commission regulation (EC) No. 208/05. Official Journal of the European Union, 2005, L34/3.

(48) Umpleby, R. J.; Baxter, S. C.; Chen, Y.; Shah, R. N.; Shimizu, K. D. Characterization of Molecularly Imprinted Polymers with the Langmuir-Freundlich Isotherm. Anal. Chem. 2001, 73, 4584-4591. 